\title{
Evidence of Assortative Mating for Theory of Mind via Facial Expressions but not Language
}

\author{
Emily Jackson ${ }^{1}$, John Galvin ${ }^{2}$, Varun Warrier ${ }^{3}$, Simon Baron-Cohen ${ }^{3}$, Shanhong Luo ${ }^{4}$, Robin \\ I. M. Dunbar ${ }^{5}$, Hannah Proctor ${ }^{1}$, Eva Lee ${ }^{1}$, and Gareth Richards ${ }^{1,3^{*}}$ \\ ${ }^{1}$ School of Psychology, Faculty of Medical Sciences, Newcastle University, UK \\ ${ }^{2}$ Department of Psychology, Birmingham City University, UK \\ ${ }^{3}$ Autism Research Centre, Department of Psychiatry, University of Cambridge, UK \\ ${ }^{4}$ Department of Psychology, University of North Carolina Wilmington, USA \\ ${ }^{5}$ Department of Experimental Psychology, University of Oxford, UK \\ * Corresponding author address: School of Psychology, Newcastle University, 4.32 Dame Margaret Barbour \\ Building, Wallace Street, Newcastle upon Tyne, UK; email: gareth.richards@ncl.ac.uk
}

\begin{abstract}
Assortative mating (AM) is a phenomenon in which romantic partners typically resemble each other at a level greater than chance. There is converging evidence that social behaviours are subject to AM, though less is known regarding social cognition. Social functioning requires the ability to identify and understand the mental states of others, i.e., Theory of Mind (ToM). The present study recruited a sample of 102 heterosexual couples via an online survey to test if ToM as measured using facial expressions (Reading the Mind in the Eyes Test) or language (Stiller-Dunbar Stories Task) is associated with AM. Results showed existence of AM for ToM via facial expressions, though no such effect for ToM via language. AM for ToM via facial expressions was not moderated by length of relationship nor by partner similarity in age, educational attainment, or religiosity, all variables relevant to social stratification. This suggests AM for ToM via facial expressions is better explained by partners being alike at the start of their relationship (initial assortment) rather than becoming similar through sustained social interaction (convergence), and by people seeking out partners that are like themselves (active assortment) rather than simply pairing with those from similar demographic backgrounds (social homogamy).
\end{abstract}

Keywords: Assortative mating; Behavioural genetics; Empathizing; Reading the Mind in the Eyes; Theory of Mind 


\section{Introduction}

Despite enduring popularity for the notion that "opposites attract", evidence from empirical research more readily supports the opposite view: that "birds of a feather flock together" (Luo, 2017; Montoya et al., 2008). In the context of romantic relationships, this reflects the presence of assortative mating (AM), that is, the non-random pattern in which partners show greater similarity to one another than expected by chance. The highest within-couple correlations are typically observed for demographic variables, such as age and ethnicity, and are followed by educational attainment and attitudes; the weakest correlations are usually reported for personality traits and physical characteristics (see Luo, 2017).

AM can arise through various means (see Luo \& Klohnen, 2005). For instance, individuals may be more likely to meet and thus form a relationship if they share a similar social background (social homogamy). However, despite the strength of within-couple correlations observed for demographic variables (Blossfeld \& Timm, 2003), this resemblance appears not to account for similarity across other characteristics. For example, AM has been reported to occur within a range of populations that are relatively demographically homogenous (e.g., Burgess \& Wallin, 1943; Godoy et al., 2008; Kardum et al., 2017; Luo, 2009; Luo \& Klohnen, 2005; Richards et al., 2020; Tognetti et al., 2014; Wu et al., 2020). AM could arise through convergence, i.e., couples may grow alike over time due to their interactive experiences and shared environment. Empirical findings cast doubt on this suggestion, as relationship length does not correlate with AM for most variables that have been measured (Kardum et al., 2017; Luo \& Klohnen, 2005; Richards et al., 2020). Furthermore, AM is detectable early in relationships (Luo, 2009), indicating the presence of initial assortment. It is thus largely accepted that couple similarity is driven mainly by initial and active assortment, rather than by social homogamy and/or convergence (Luo \& Klohnen, 2005).

Social abilities may play an important role in AM, as they could not only affect initial pairing but also influence interactions throughout a relationship. Indeed, there is some evidence for $\mathrm{AM}$ in social skills and characteristics related to interpersonal functioning. For instance, following the AM theory of autism (Baron-Cohen, 2006a, 2006b), a number of studies report AM effects for traits associated with autism, a spectrum of neurodevelopmental conditions characterised in part by difficulties in social interaction (Connolly et al., 2019; Constantino \& Todd, 2005; Richards et al., 2020; Virkud et al., 2009). Moreover, Burleson and Denton (1992) 
found evidence of AM for cognitive complexity, a socio-cognitive construct that enables social information processing and facilitates perspective taking (Hale \& Delia, 1976). Interestingly, couples consisting of two low-scoring partners were no less satisfied than couples consisting of two high-scoring partners. The authors therefore suggest that similarity in social abilities can promote enjoyable and harmonious interactions that ultimately enhance relationship satisfaction. Such assortment is also evident in platonic relationships, with friends showing increased similarity for relevant skills such as communication ability (Burleson et al., 1992) and empathic distress (Smith \& Rose, 2011), as well as for measures of autistic traits (Faso et al., 2016; Wainer et al., 2013). These findings suggest that individuals seek relationships with others who are cognitively similar. This could be because comparable outlooks that validate one's own beliefs are positively reinforcing (Burleson et al., 1992; Montoya et al., 2008). Alternatively, such effects could be a passive product of mating market operation (Luo, 2017). Essentially, as the most desirable partners are likely to be those with high levels of social skills, competition for such partners will be most intense. This could result in those with high levels of social skills being more likely than chance to pair with each other, leaving those with lower levels of social skills to choose partners from the remaining pool of potential mates characterised by lower social skills.

Couple assortment for social abilities may rely to some degree on a capacity to interpret the mind states of others and to understand that these can differ from one's own mind state: that is, it relies on a 'Theory of Mind' (ToM) (Premack \& Woodruff, 1978; Baron-Cohen, 1995). Two dissociable yet related components of ToM have been identified: socio-cognitive and socio-perceptual (Dvash \& Shamay-Tsoory, 2014; Shamay-Tsoory \& Aharon-Peretz, 2007; Tager-Flusberg \& Sullivan, 2000). The socio-cognitive component encompasses the ability to reason about the mental states of others (Nettle \& Liddle, 2008). It is commonly assessed by measures such as the Stiller-Dunbar Stories Task (Powell et al., 2010; Stiller \& Dunbar, 2007), which require participants to read socially complex short stories and then answer questions about the perspectives of characters presented within them. The socio-perceptual component of ToM, on the other hand, relates to the ability to rapidly detect the mental states of others based on peripheral cues such as facial expressions and body movements (Nettle \& Liddle, 2008). It is commonly measured using the Reading the Mind in the Eyes Test (RMET; BaronCohen et al., 2001), which requires participants to identify mental states from images of the eye region of people's faces. Socio-perceptual ToM therefore appears to represent the more reflexive component, and is closely related to the affective system (see Tager-Flusberg \& 
Sullivan, 2000). Notably, there is also evidence for distinct 'nice' and 'nasty' ToM (Ronald et al., 2005), and one's ability in each domain may be differentially associated with prosocial and antisocial outcomes (Lonigro et al., 2014). The terms 'perceptual' and 'cognitive' are not ideal in the current context since these are not easy to disentangle because the former entails the latter. Therefore, in what follows, we use different terminology that we hope is more descriptive and unambiguous: we refer to the RMET as a test of ToM via facial expressions and the Stiller-Dunbar Stories Task as a test of ToM via language.

Richards et al. (2020) reported preliminary evidence of AM for ToM via facial expressions, with scores on the RMET being positively correlated within heterosexual couples from the UK. However, the sample size was relatively small ( $\mathrm{n}=55$ couples), the finding has not yet been independently replicated, and the study did not examine ToM via language. Nevertheless, as there is evidence for AM in relation to constructs closely related to ToM via language, such as cognitive complexity (Burleson \& Denton, 1992) and emotional intelligence (Śmieja \& Stolarski, 2018), and because partner similarity in such characteristics appears advantageous for the formation and maintenance of interpersonal relationships, positive AM can be predicted to occur.

The current study was pre-registered on the Open Science Framework (osf.io/bk5c3) and aimed to investigate within-couple similarity for ToM. We predicted that (1) total scores for RMET and the Stiller-Dunbar Stories Task measures would each be positively correlated between male and female partners in heterosexual relationships, and (2) that smaller difference scores (indicating increased similarity) for these variables would be observed for actual couples than for random male-female pairings. Additionally, we predicted that any couple similarity effects observed would reflect initial and active assortment rather than convergence and/or social homogamy.

\section{Method}

\subsection{Participants}

We conducted an a priori power analysis using G*Power 3.1 (Faul et al., 2007, 2009) to determine the required sample size. Using the effect size estimate of within-couple correlation for the RMET previously reported by Richards et al. (2020) ( $r=0.438)$, and 80\% power, a sample size of $\mathrm{n}=38$ couples would be required to observe a statistically significant effect $(p<$ 
0.05) using a two-tailed Pearson's correlation. However, we aimed to collect data from $n=100$ couples because (1) we intended to negate the loss of statistical power associated with missing data (i.e., some participants might not complete all required measures), (2) we aimed to protect against 'winner's curse' and effect size inflation associated with relatively small sample sizes, and (3) because the dataset was additionally used to examine AM in relation to Dark Triad personality traits, which have been reported to show a smaller within-couple correlation (results from this additional study were also pre-registered and will be presented separately). Ethical approval was obtained from the Faculty of xxxx Ethics Committee, xxxx University (reference number: 7573/2020).

Participants comprised an opportunity sample of heterosexual couples recruited through word of mouth and snowball sampling. To take part in the study, participants were required to be aged 18 years or over and to be in a heterosexual relationship at the time. Data were available for $n=104$ complete couples (i.e., those couples for whom both partners completed at least one of the ToM measures), although two couples that each included one non-binary member were not included in further analysis. As participants were asked to specify their sex (not gender identity), these couples were removed because it is difficult to determine whether this item was interpreted correctly. For those $n=102$ couples that were retained, the mean age of males was $36.64(S D=17.83)$, and that for females was $M=35.55(S D=17.62)$. The mean length of relationship was $12.91(S D=14.99)$, and most participants were UK residents (males = 95.96\%; females $=96.04 \%$ ). The majority (males: 63.73\%; females: 56.86\%) were employed and of White/Caucasian ethnicity (males: $n=89,87.25 \%$; females: $n=86,84.31 \%$ ), and a little over one third were students (males: 32.35\%; females: 43.14\%). Slightly more than half (55.88\%) reported living with their partner; approximately one third were married (males: $n=38,37.26 \%$; females: $n=39,38.24 \%$ ), few were engaged (males: $n=2,1.96 \%$; females, $n=2$, $1.96 \%$ ), and most were not married (males: $n=62,60.78 \%$; females: $n=61,59.80 \%$ ). It is unclear why 39 females, but only 38 males, reported being married. Although speculative, this could reflect one of the females remaining legally married to a previous partner from whom she had separated. The sample as a whole reported relatively high academic attainment, as follows: no qualifications (males: $n=2,1.96 \%$; females: $n=0,0 \%$ ); completed GCSE level (or equivalent) (males: $n=9,8.82 \%$; females, $n=8,7.84 \%$ ); completed A level, Access Course (or equivalent) (males: $n=40,39.22 \%$; females: $n=42,41.18 \%$ ); Bachelor's Degree (males: $n=38$, 37.26\%; females: $n=33,32.35 \%$ ); Master's Degree (males: $n=9,8.82 \%$; females: $n=16$, 15.69\%); Doctorate Degree (males: $n=4,3.92 \%$; females: $n=3,2.94 \%$ ). 


\subsection{Design}

The present study employed a correlational design to examine within-couple correlations/similarity for measures of RMET (ToM via facial expressions) and the StillerDunbar Stories Task (ToM via language).

\subsection{Materials}

Participants were administered demographic questions to determine their age, sex, country of residence, ethnicity, educational attainment, living status (i.e., cohabiting with partner or not), relationship length, and marital status. As large social homogamy effects may occur in regard to religious beliefs and practices, we also used the General Religiosity Measure (Saroglou \& Galand, 2004; Saroglou \& Muñoz-García, 2008). This provides a 3-item indication of intrinsic ('general') religiosity, i.e., the strength of a person's religious beliefs or feelings and how important those beliefs are in their life, regardless of which religion they may (or may not) follow. Each item is answered on a 7-point Likert scale, with higher scores indicating higher religiosity. Cronbach's alpha for the present study indicated satisfactory internal consistency $(\alpha=0.752)$.

We used a revised version of Stiller and Dunbar's (2007) Stories Task, similar to that of Powell et al. (2010), to measure ToM via language. This comprises five short stories, each approximately 200 words long, which depict various social scenarios involving interactions between three to five characters. After reading each story, participants were asked to answer true or false questions relating to its content. These assessed the ability to consider various levels of perspectives ranging from two to six levels of intentionality, where two requires the participant to track the perspective of one character and six requires the participant to track five embedded levels of perspective. Factual questions used in Stiller and Dunbar's (2007) original study were omitted here to reduce the length of the questionnaire and because we did not set out to investigate memory ability. Participants were advised to answer as quickly as possible and to refrain from changing their answers once selected. A total score was calculated from the number of correct responses and could range from 0 to 49. Cronbach's alpha indicated satisfactory internal consistency $(\alpha=0.601)$.

To test ToM via facial expressions, we used the Reading the Mind in the Eyes Test (RMET) (Baron-Cohen et al., 2001). The RMET assesses the ability to correctly identify mental states 
from pictures of the eye region of people's faces. More specifically, participants were shown 36 images of eyes (18 male and 18 female) portraying various expressions. Each image was presented individually along with four adjectives (one target word and three foil words) describing complex mental states (e.g., contemplative, encouraging, despondent, flustered). Participants were instructed to select the adjective they believed best described the mental state depicted in each picture (a unique set of adjectives was included for every image). They also had access to dictionary definitions of the adjectives and were advised to respond based on instinct and to refrain from changing any answers once they had been selected. A total score was calculated as the number of correct responses across 36 trials. Internal consistency for the RMET in the present study was satisfactory $(\alpha=0.713)$.

\subsection{Procedure}

Participants over 18 years of age and known to be in a heterosexual relationship were contacted by the researchers and asked to participate in an online survey (hosted by Qualtrics) investigating cognitive and perceptual abilities of romantic partners. They were given information about the study, asked to provide consent before proceeding further, and were debriefed on completion. Participants were advised to complete the survey independently, in one sitting, and to avoid discussing any of the tasks with their partner during the study. They were also offered an opportunity to enter a prize draw for a $£ 25$ Amazon voucher.

\subsection{Data Analysis}

Paired samples $t$ tests were conducted to test for within-couple sex differences in the ToM measures. We then used Pearson's tests to examine within couple correlations for variables relevant to social homogamy (age, intrinsic religiosity, and educational attainment [note that, due to the ordinal coding of the educational attainment variable, a Spearman's test was used in this case]).

We conducted both variable-centred and couple-centred analyses to examine within-couple correlation/similarity for ToM variables (see Luo \& Klohnen, 2005). For the variable-centred analysis, we used Pearson's tests to determine the strength and direction of correlation within couples for the Stiller-Dunbar Stories Task total score and RMET total score. To conduct the couple-centred analysis, we first computed unsigned difference scores (for both the RMET and Stiller-Dunbar Stories Task, respectively) for each couple by subtracting the standardised score 
of the female from the standardised score of the male. We next computed difference scores for random pairings by matching every male with every female in the dataset that was not his partner (and therefore also matching every female with every male in the dataset that was not her partner) and taking the average. We used paired-samples $t$ tests to compare the unsigned difference scores for actual couples with those computed for random pairings. Smaller difference scores for actual couples than random couples indicate a greater level of partner resemblance than expected under conditions of random mating.

If a statistically significant (i.e., $p<0.05$, two-tailed) within couple-correlation (i.e., from the couple-centred approach) was detected for a ToM measure, we correlated the within-couple (unsigned) difference score for that measure with the length of relationship. The idea here is that if smaller difference scores (i.e., greater similarity) are associated with longer relationships (indicated by a significant negative correlation) then partner resemblance may be better explained by convergence than initial assortment. We also correlated the unsigned withincouple difference score for the ToM measure with the within-couple difference scores for three variables relevant to social homogamy (Luo \& Klohnen, 2005): age, educational attainment, and intrinsic religiosity. In this case, a significant positive correlation would indicate that couples which are similar in their demographic background are more similar for ToM than expected by chance, and so would provide evidence of social homogamy rather than active assortment.

Data analysis was conducted in RStudio (Version 1.3.1073), and all tests were two-tailed. A criterion of $p<0.05$ was used to indicate statistical significance, and effect sizes were interpreted in accordance with generally accepted criteria (Cohen, 1988). The dataset and R code used to run the analysis are available on the Open Science Framework (osf.io/bk5c3).

\section{Results}

\subsection{Sex Differences}

Paired samples $t$ tests revealed that females scored significantly higher than their male partners on both the RMET and the Stiller-Dunbar Stories Task (Table 1) as previously reported (Baron-Cohen et al., 2015; Stiller \& Dunbar, 2007). 
Table 1. Descriptive statistics and sex differences for Theory of Mind variables.

\begin{tabular}{lllllllllll} 
& Males & \multicolumn{6}{c}{ Females } & \multicolumn{2}{c}{ Difference } \\
& $\boldsymbol{M}$ & $\boldsymbol{S D}$ & $\boldsymbol{M}$ & $\boldsymbol{S D}$ & $\boldsymbol{t}$ & $\boldsymbol{d} \boldsymbol{f}$ & $\boldsymbol{p}$ & $\boldsymbol{d}$ \\
\hline RMET & & & & & & & & \\
Stiller-Dunbar Stories Task & 35.19 & 4.23 & 37.54 & 3.75 & -4.284 & 99 & $<0.001$ & -0.591 \\
\hline
\end{tabular}

Note. $\mathrm{RMET}=$ Reading the Mind in the Eyes Test. $M=$ mean. $S D=$ standard deviation.

\subsection{Variable-centred analysis}

A strong positive correlation was observed between partners' ages, $r(100)=0.980, p<.001$, and moderate within-couple correlations were present for educational attainment $r_{s}=0.426, p$ $<0.001$, and intrinsic religiosity, $r(100)=0.445, p<0.001$. As predicted, partners' scores on the RMET were significantly positively correlated, $r(94)=0.284, p=0.005$ (Figure 1a); however, there was no such effect for the Stiller-Dunbar Stories Task, $r(98)=0.048, p=0.635$ (Figure 1b). Although not specified in our pre-registered analysis plan, we used Pearson's correlations to examine the relationship between ToM via facial expressions and ToM via language. The RMET and Stiller-Dunbar Stories Task were significantly and moderately positively correlated in both males, $r(95)=0.262, p=0.010$, and females, $r(97)=0.461, p<$ 0.001. A Fisher's $r$-to- $z$ test showed that the difference in strength for these correlations was not statistically significant, $z=-1.59, p=0.112$ (two-tailed).

\subsection{Couple-centred analysis}

The unsigned difference score for the RMET was smaller for actual couples $(M=0.944, S D=$ 0.73) than the average calculated from all other male-female pairings possible within the dataset $(M=1.096, S D=0.426), t(95)=-2.218, p=0.029, d=-0.243$ (see Figure 2 for violin boxplots). In accord with findings from the variable-centred analysis, within-couple difference scores for the Stiller-Dunbar Stories Task were not significantly different (actual couples: $M=$ $1.088, S D=0.830$; average of other male-female pairings: $M=1.096, S D=0.407), t(99)=$ $0.107, p=0.915, d=-0.011$. 


\subsection{Initial assortment vs. convergence, and active assortment vs. social homogamy}

As a significant within-couple correlation was observed for the RMET, we correlated the unsigned within-couple difference scores for this variable with length of relationship to test for evidence of a convergence effect. No significant correlation emerged, $r(94)=-0.085, p=0.408$, suggesting that couple similarity for this variable is better explained by initial assortment. To test for social homogamy, we then correlated the unsigned within-couple difference scores for the RMET with the unsigned within-couple difference scores for age, educational attainment, and intrinsic religiosity. No significant effects were observed (age: $r[94]=-0.039, p=0.707$; educational attainment, $r[94]=0.127, p=0.219$; intrinsic religiosity, $r[94]=-0.052, p=0.616$ ), suggesting that couple resemblance for this measure is better explained by active assortment.

Figure 1. Scatterplots showing within-couple correlations for (a) RMET and (b) Stiller-Dunbar Stories Task measures of ToM ability.
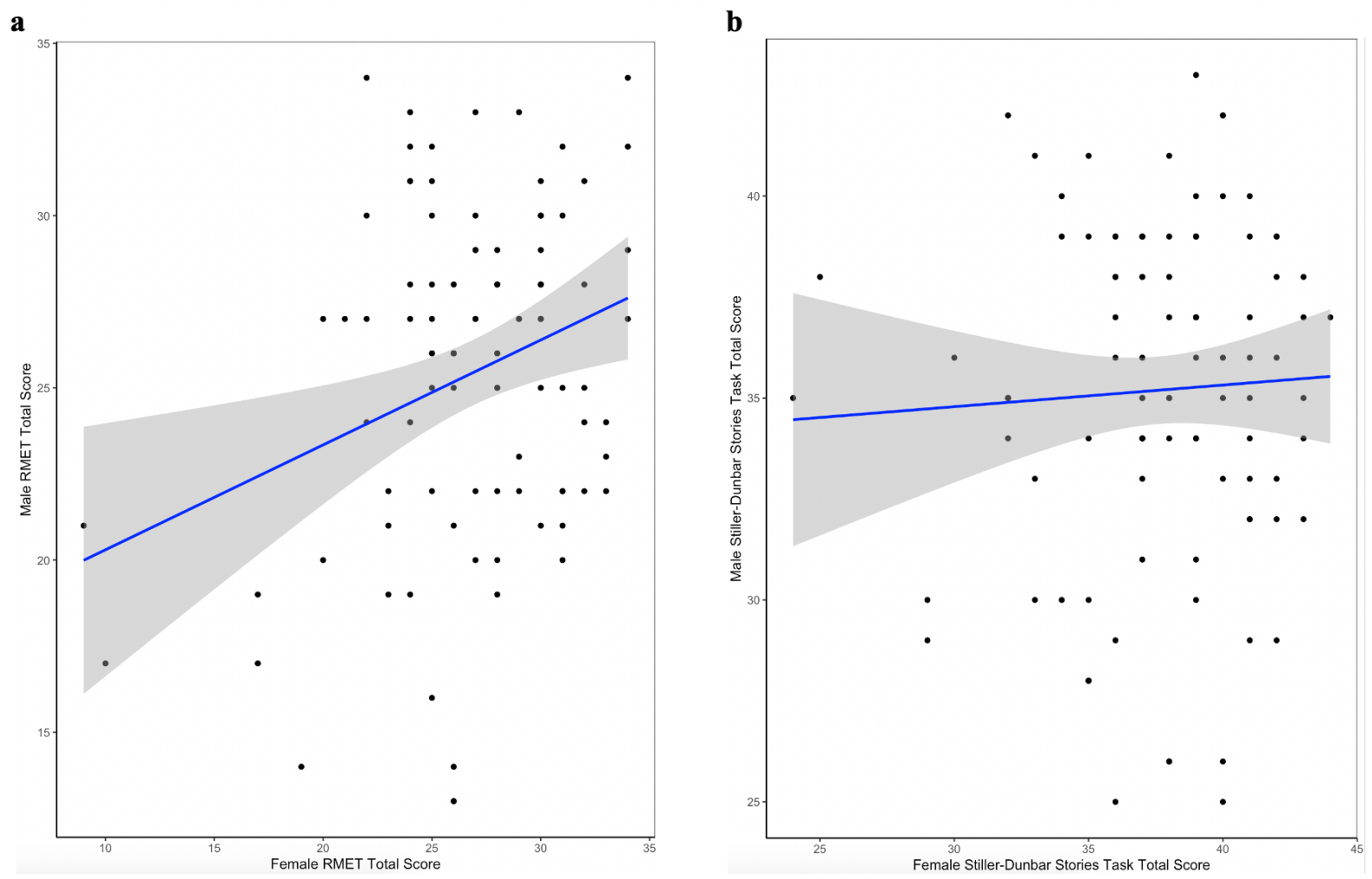
Figure 2. Violin boxplots showing the distribution of unsigned difference scores for the RMET for (a) actual couples and (b) the average of all other male-female pairings possible within the dataset.

a

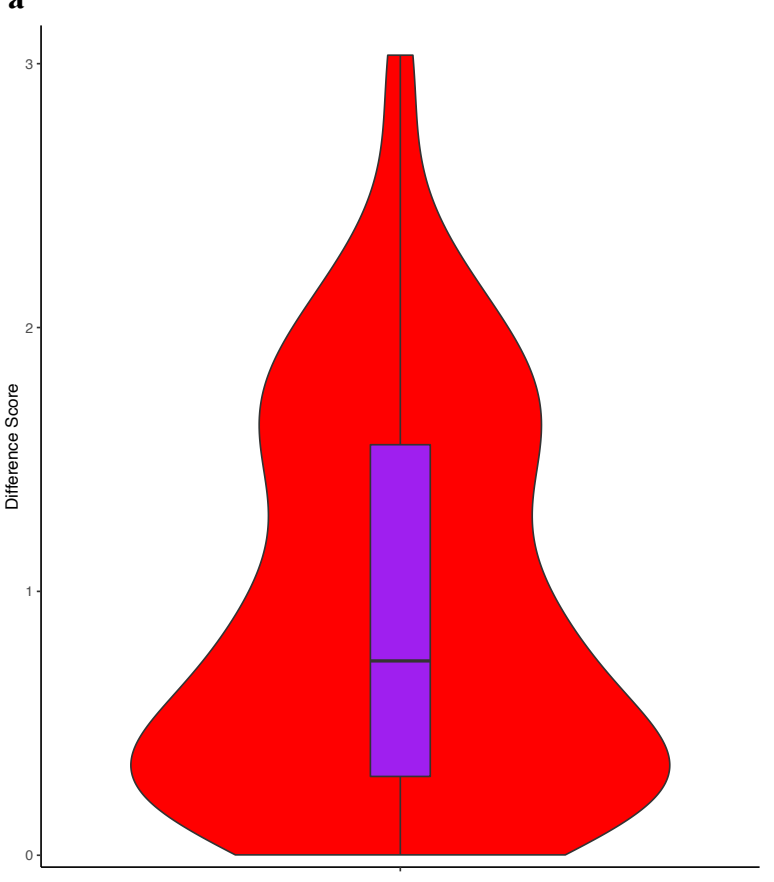

Actual Couples b

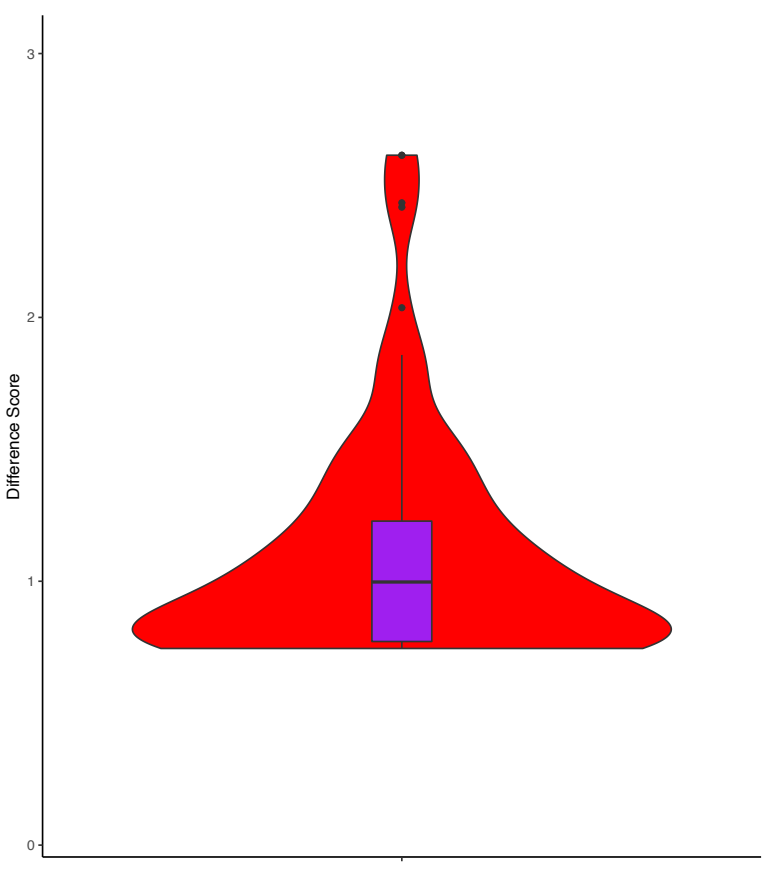

Average of Random Male-Female Pairings 


\section{Discussion}

The present study aimed to examine couple similarity for Theory of Mind (ToM) via facial expressions vs. language. In line with our hypothesis, ToM ability as measured via the Reading the Mind in the Eyes Test (RMET) showed a positive correlation between partners. We also observed that actual couples were more alike for this variable (i.e., they had smaller difference scores) compared to the average of all other male-female pairings possible within the dataset. Further analysis revealed that the within-couple correlation for ToM via facial expressions was not moderated by relationship length nor by partner similarity in age, educational attainment, or intrinsic religiosity, which suggests that the association is better explained by initial and active assortment than by convergence or social homogamy. Although these effects were in accord with our predictions, we did not observe any evidence of assortative mating for ToM via language, as measured via the Stiller-Dunbar Stories Task.

The positive correlation observed between partners' ToM ability via facial expressions directly replicates the earlier finding of Richards et al. (2020); more specifically, both studies reported evidence from variable-centred and couple-centred analyses for increased couple similarity in performance on the RMET. Furthermore, these studies found that the within-couple correlation for this measure was better explained by initial and active assortment than by convergence or social homogamy. As Richards et al. (2020) administered the RMET in a lab study whereas here we utilised online survey methodology, the effect appears to be replicable across settings. These findings also align with wider research in which positive assortment has been reported for characteristics related to socio-perceptual ToM ability (e.g., Kardum et al., 2017; Śmieja \& Stolarski, 2018).

When considering why positive assortment occurs for ToM via facial expressions, it is possible that similarity in social abilities leads to more satisfying relationships. For example, those who perceive themselves as having similar levels of empathy (Kimmes et al., 2014) and emotional intelligence (Smith et al., 2008) as their partners report higher relationship satisfaction than those who perceive themselves as being disparate in these domains. Additionally, ToM ability via facial expressions is positively correlated with prosocial orientation (Declerck \& Bogaert, 2008). This is notable because prosocial behaviours exhibit spousal resemblance (Tognetti et al., 2014) and are likely beneficial in terms of relationship and reproductive outcomes (ibid). Moreover, similarity in ToM ability via facial expressions may allow for continual validation 
throughout the course of a relationship. Such validation can enhance feelings of intimacy between partners (Reis \& Shaver, 1988), which in turn may underpin the finding that similarity in empathic social abilities predicts relationship satisfaction (Kimmes et al., 2014; Smith et al., 2008). Additionally, couple similarity in general may be of benefit at the emotional level, as it is associated with relatively high life satisfaction both of parents and their children (Wu et al., 2020; though cf. Godoy et al., 2008). Thus, AM for ToM via facial expressions (and indeed AM more generally) may act as a fitness-enhancing mechanism that promotes reproductive success. To test this idea, future research should investigate whether couple similarity in ToM ability is predictive of relationship outcomes (e.g., relationship satisfaction) and reproductive fitness (e.g., number of offspring).

Turning to ToM via language, the lack of partner correlation observed in the current study is at odds with previous research reporting evidence of assortment for closely related abilities (Burleson \& Denton, 1992; Constantino \& Todd, 2005; Richards et al., 2020; Śmieja \& Stolarski, 2018). Furthermore, as ToM via facial expressions and language were moderately positively correlated, and ToM via facial expressions showed evidence of positive assortment, we might expect ToM via language to show a similar pattern simply due to it being a facet of the same underlying construct (i.e., ToM as a whole). While these contrasting findings support the notion that these two aspects of ToM are related yet dissociable abilities (Dvash \& ShamayTsoory, 2014; Shamay-Tsoory \& Aharon-Peretz, 2007; Tager-Flusberg \& Sullivan, 2000), they contradict our hypothesis because we expected both to show evidence of AM.

Taking the above observations into account, it may be important to consider the nature of abilities. Abilities are internal attributes, meaning for assortment to occur, the ability must be expressed externally in a consistent and reliable way. Indeed, it has been suggested that withincouple correlations for such variables may be relatively small simply due to the difficulties inherent with accurately assessing them (Śmieja \& Stolarski, 2018). It is also worth considering that ToM via facial expressions and language rely on distinct neural substrates (Dvash \& Shamay-Tsoory, 2014). This makes it noteworthy that Smith \& Rose (2011) reported empathic distress (an affective response) but not social perspective-taking to be significantly positively correlated within early-adolescent friendship dyads, a pattern of findings that somewhat mirrors that of the present study. The connection between ToM via facial expressions and the neural substrates involved in emotional processing (Dvash \& Shamay-Tsoory, 2014) could also suggest that relevant behavioural practices increase visibility of this skill more effectively than 
is possible for ToM via language. Thus, self-resemblance in this ability may be more easily detected and evaluated by potential partners, making it more likely to undergo AM.

A further consideration is the types of behaviour that are associated with abilities. For instance, while both ToM components are positively correlated with prosocial behaviour in children (Imuta et al., 2016), differences emerge when considering their relation to antisocial tendencies. Sutton et al. (1999) found 'socio-cognitive' ToM to correlate positively with bullying behaviours, suggesting that an ability to understand the mind states of others may give rise to the capacity for manipulative aggression. 'Socio-perceptual' ToM, on the other hand, has been found to correlate negatively with conduct problems (Sharp, 2008). Machiavellianism, a personality trait characterised by manipulative behaviour and a lack of interpersonal affect, is also negatively correlated with socio-perceptual ToM in young adults (Ali \& Charmorro-Premuzic, 2010), but is not characterised by deficits in socio-cognitive ToM (Paal \& Bereczkei, 2007). Thus, it appears that the socio-perceptual component of ToM, likely due to its links with affect and empathy (Eisenberg \& Miller, 1987), more consistently predicts prosocial behaviour and a lack of antisocial behaviour, whereas socio-cognitive ToM correlates positively with both of these. Furthermore, different facets of ToM (e.g., 'nice' and 'nasty' ToM; Ronald et al., 2005) may relate differently to prosocial and antisocial behaviour (Lonigro et al., 2014). Such added complexity could make detecting couple assortment for overall sociocognitive ToM difficult, as it is not reliably or consistently expressed.

It should be noted that the Stiller-Dunbar Stories Task is designed to assess the upper limit of an individual's ability (Stiller \& Dunbar, 2007); thus, if an individual's maximal ToM via language does not reliably reflect the ability level displayed throughout daily life, it is unlikely that assortment for maximal ability will occur (unless as a by-product of assortment for some other related trait). Importantly, Keysar et al. (2003) found that adults do not routinely use the full extent of their socio-cognitive ToM ability, and so we may not have correlated the level at which partners' socio-cognitive ToM abilities are actually used throughout daily life. This may explain why the present findings differ from those of previous studies. For example, Burleson and Denton (1992) found evidence of AM for cognitive complexity, an ability closely related to socio-cognitive ToM (Hale \& Delia, 1976), when this was assessed based on participants' written descriptions of peers. Thus, rather than maximal ability, this task likely assesses individuals' natural level of cognitive complexity, and so the level that is expected to be most visible to others. Similarly, couple-similarity has been observed for abilities closely related to 
socio-cognitive ToM when assessed via spouse-report (Constantino \& Todd, 2005; Smith et al., 2008). While spouse estimations may not accurately reflect underlying ability, they are likely based on the ability level that is evident through partners' behaviour. Thus, even if partners are not matched in their maximal socio-cognitive ToM ability, they may be similar in the way and extent to which this ability is typically deployed. Future research should therefore investigate couple assortment for the level at which socio-cognitive ToM is most routinely used.

Although there are notable strengths to the current research, such as the pre-registered hypothesis and analysis plan, as well as the sample size having been determined via a priori power calculation, there are also some limitations that should be acknowledged. First, although participants were asked not to confer with their partner when completing the study, the online nature of the research made it impossible to verify whether this request was followed. However, as conferring might be expected to increase rather than decrease similarity in responding, this appears unlikely to have been a problem. That is because the within-couple correlation for the RMET observed here $(r=0.284)$ was actually smaller than that of the lab-based study of Richards et al. (2020) $(r=0.438)$ which used the same measure. Nevertheless, the current study is limited in that the sample predominantly represents Western, Educated, Industrialised, Rich, and Democratic (WEIRD) individuals/societies, and so may not be generalisable to the human condition writ large (Henrich et al., 2010). This is particularly relevant in the context of AM, as for most of evolutionary history, homo sapiens has lived in relatively small and homogeneous groups in which marital pairings would often be initiated to forge/maintain political alliances rather than due to the active preferences of those involved (Chagnon, 1983). In the developed world, however, such processes are less common, and most people live in large and heterogeneous settlements, and so have access to a vastly increased pool of potential mates. The rise of internet dating has also facilitated active assortment (Lee, 2016), meaning that findings from studies of WEIRD populations, albeit informative regarding mate selection in modern humans, may not be an accurate reflection of our evolutionary past. Future research should therefore aim to examine similarity/dissimilarity of AM processes within pre-industrial societies (Godoy et al., 2008).

\section{Conclusions}


The current study provides evidence consistent with there being positive assortative mating for ToM via facial expression but not for ToM via language. We suggest that inherent differences in the way these abilities are externally expressed may explain the contrasting findings. It could be that the level of this skill most routinely displayed during daily life is not estimated accurately by measures designed to determine a person's maximal ability. Further research is therefore required to explore these possibilities, and to determine whether assortative mating occurs for different aspects of ToM within non-WEIRD populations.

\section{Additional information}

\section{Competing Interests}

The authors declare no competing interests.

\section{Funding}

SBC received funding from the Wellcome Trust $214322 \backslash Z \backslash 18 \backslash Z$. For the purpose of Open Access, the author has applied a CC BY public copyright licence to any Author Accepted Manuscript version arising from this submission. Further to this SBC received funding from Innovative Medicines Initiative 2 Joint Undertaking (JU) under grant agreement No 777394. The JU receives support from the European Union's Horizon 2020 research and innovation programme and EFPIA and AUTISM SPEAKS, Autistica, SFARI. SBC also received funding from the Autism Research Trust, Autistica, the MRC and the NIHR Cambridge Biomedical Research Centre. The research was supported by the National Institute for Health Research (NIHR) Collaboration for Leadership in Applied Health Research and Care East of England at Cambridgeshire and Peterborough NHS Foundation Trust. VW was funded by the Autism Research Trust, the Wellcome Trust, Templeton World Charity Inc., and the NIHR Biomedical Research Centre in Cambridge, during the period of this work. The views expressed are those of the authors, and not necessarily those of the NIHR, NHS or Department of Health and Social Care.

\section{Author contributions}

GR, EJ, SBC, SL, RB, JG, and VW designed the study; EJ, HP, and EL collected the research data; GR performed data analysis; EJ and GR wrote the first draft, and all authors read and approved the final manuscript prior to submission. 


\section{Data accessibility statement}

The dataset and $\mathrm{R}$ code used to run the analyses in this paper are available on the Open Science Framework (osf.io/bk5c3).

\section{References}

Baron-Cohen, S. (1995). Mindblindness: An essay on autism and theory of mind. MIT Press. Baron-Cohen, S. (2006a). The hyper-systemizing, assortative mating theory of autism. Progress in Neuro-Psychopharmacology and Biological Psychiatry, 30(5), 865-872. https://doi.org/10.1016/j.pnpbp.2006.01.010

Baron-Cohen, S. (2006b). Two new theories of autism: Hyper-systemising and assortative mating. Archives of Disease in Childhood, 91(1), 2-5. https://doi.org/10.1136/adc.2005.075846

Baron-Cohen, S., Bowen, D. C., Holt, R. J., Allison, C., Auyeung, B., Lombardo, M. V., Smith, P., \& Lai, M.-C. (2015). The "Reading the Mind in the Eyes" test: Complete absence of typical sex difference in $\sim 400$ men and women with autism. PLOS ONE, 10(8), e0136521. https://doi.org/10.1371/journal.pone.0136521

Baron-Cohen, S., Wheelwright, S., Hill, J., Raste, Y., \& Plumb, I. (2001). The "“Reading the Mind in the Eyes"' test revised version: A study with normal adults, and adults with Asperger syndrome or high-functioning autism. Journal of Child Psychology and Psychiatry, 42(2), 241-251. https://doi.org/10.1111/1469-7610.00715

Blossfeld, H.-P., \& Timm, A. (Eds.). (2003). Who marries whom? Educational systems as marriage markets in modern societies. Kluwer Academic Publishers.

Burgess, E. W., \& Wallin, P. (1943). Homogamy in social characteristics. American Journal of Sociology, 49(2), 109-124. https://doi.org/10.1086/219346

Burleson, B. R., \& Denton, W. H. (1992). A new look at similarity and attraction in marriage: Similarities in social-cognitive and communication skills as predictors of attraction and satisfaction. Communications Monographs, 59(3), 268-287. https://doi.org/10.1080/03637759209376269

Burleson, B. R., Samter, W., \& Lucchetti, A. E. (1992). Similarity in communication values as a predictor of friendship choices: Studies of friends and best friends. Southern Journal of Communication, 57(4), 260-276. https://doi.org/10.1080/10417949209372873

Chagnon, N. A. (1983). Yạomamö: The fierce people (3rd ed.). Holt, Rinehart and Winston, Inc.

Cohen, J. (1988). Statistical power analysis for the behavioral sciences (2nd ed.). Lawrence Erlbaum Associates.

Connolly, S., Anney, R., Gallagher, L., \& Heron, E. A. (2019). Evidence of assortative mating in autism spectrum disorder. Biological Psychiatry, 86(4), 286-293. https://doi.org/10.1016/j.biopsych.2019.04.014

Constantino, J. N., \& Todd, R. D. (2005). Intergenerational transmission of subthreshold autistic traits in the general population. Biological Psychiatry, 57(6), 655-660. https://doi.org/10.1016/j.biopsych.2004.12.014

Declerck, C. H., \& Bogaert, S. (2008). Social value orientation: Related to empathy and the ability to read the mind in the eyes. Journal of Social Psychology, 148(6), 711-726. https://doi.org/10.3200/SOCP.148.6.711-726

Dvash, J., \& Shamay-Tsoory, S. G. (2014). Theory of mind and empathy as multidimensional 
constructs: Neurological foundations. Topics in Language Disorders, 34(4), 282-295. https://doi.org/10.1097/TLD.0000000000000040

Eisenberg, N., \& Miller, P. A. (1987). The relation of empathy to prosocial and related behaviors. Psychological Bulletin, 101(1), 91-119. https://doi.org/10.1037/00332909.101.1.91

Faso, D. J., Corretti, C. A., Ackerman, R. A., \& Sasson, N. J. (2016). The broad autism phenotype predicts relationship outcomes in newly formed college roommates. Autism, 20(4), 412-424. https://doi.org/10.1177/1362361315585733

Faul, F., Erdfelder, E., Buchner, A., \& Lang, A.-G. (2009). Statistical power analyses using $\mathrm{G}^{*}$ Power 3.1: Tests for correlation and regression analyses. Behavior Research Methods, 41(4), 1149-1160. https://doi.org/10.3758/BRM.41.4.1149

Faul, F., Erdfelder, E., Lang, A.-G., \& Buchner, A. (2007). G*Power 3: A flexible statistical power analysis program for the social, behavioral, and biomedical sciences. Behavior Research Methods, 39(2), 175-191. https://doi.org/10.3758/bf03193146

Godoy, R., Eisenberg, D. T. A., Reyes-García, V., Huanca, T., Leonard, W. R., McDade, T. W., Tanner, S., \& TAPS Bolivian Research Team. (2008). Assortative mating and offspring well-being: Theory and empirical findings from a native Amazonian society in Bolivia. Evolution and Human Behavior, 29(3), 201-210. https://doi.org/10.1016/j.evolhumbehav.2007.12.003

Hale, C. L., \& Delia, J. G. (1976). Cognitive complexity and social perspective-taking. Communications Monographs, 43(3), 195-203. https://doi.org/10.1080/03637757609375932

Henrich, J., Heine, S. J., \& Norenzayan, A. (2010). The weirdest people in the world? Behavioral and Brain Sciences, 33(2-3), 61-83. https://doi.org/10.1017/s0140525x0999152x

Imuta, K., Henry, J. D., Slaughter, V., Selcuk, B., \& Ruffman, T. (2016). Theory of mind and prosocial behavior in childhood: A meta-analytic review. Developmental Psychology, 52(8), 1192-1205. https://doi.org/10.1037/dev0000140

Kardum, I., Hudek-Knezevic, J., Schmitt, D. P., \& Covic, M. (2017). Assortative mating for Dark Triad: Evidence of positive, initial, and active assortment. Personal Relationships, 24(1), 75-83. https://doi.org/10.1111/pere.12168

Keysar, B., Lin, S., \& Barr, D. J. (2003). Limits on theory of mind use in adults. Cognition, 89(1), 25-41. https://doi.org/10.1016/S0010-0277(03)00064-7

Kimmes, J. G., Edwards, A. B., Wetchler, J. L., \& Bercik, J. (2014). Self and other ratings of dyadic empathy as predictors of relationship satisfaction. American Journal of Family Therapy, 42(5), 426-437. https://doi.org/10.1080/01926187.2014.925374

Kurdek, L. A., \& Krile, D. (1982). A developmental analysis of the relation between peer acceptance and both interpersonal understanding and perceived social self-competence. Child Development, 53(6), 1485-1491. https://doi.org/10.2307/1130075

Lee, S. (2016). Effect of online dating on assortative mating: Evidence from South Korea. Journal of Applied Econometrics, 31(6), 1120-1139. https://doi.org/10.1002/jae.2480

Lonigro, A., Laghi, F., Baiocco, R., \& Baumgartner, E. (2014). Mind reading skills and empathy: Evidence for nice and nasty ToM behaviours in school-aged children. Journal of Child and Family Studies, 23(3), 581-590. https://doi.org/10.1007/s10826-013-97225

Luo, S. (2009). Partner selection and relationship satisfaction in early dating couples: The role of couple similarity. Personality and Individual Differences, 47(2), 133-138. https://doi.org/10.1016/j.paid.2009.02.012

Luo, S. (2017). Assortative mating and couple similarity: Patterns, mechanisms, and consequences. Social and Personality Psychology Compass, 11(8), e12337. 
https://doi.org/10.1111/spc3.12337

Luo, S., \& Klohnen, E. C. (2005). Assortative mating and marital quality in newlyweds: A couple-centered approach. Journal of Personality and Social Psychology, 88(2), 304326. https://doi.org/10.1037/0022-3514.88.2.304

Montoya, R. M., Horton, R. S., \& Kirchner, J. (2008). Is actual similarity necessary for attraction? A meta-analysis of actual and perceived similarity. Journal of Social and Personal Relationships, 25(6), 889-922. https://doi.org/10.1177/0265407508096700

Nettle, D., \& Liddle, B. (2008). Agreeableness is related to social-cognitive, but not socialperceptual, theory of mind. European Journal of Personality, 22(4), 323-335. https://doi.org/10.1002/per.672

Paal, T., \& Bereczkei, T. (2007). Adult theory of mind, cooperation, Machiavellianism: The effect of mindreading on social relations. Personality and Individual Differences, 43(3), 541-551. https://doi.org/10.1016/j.paid.2006.12.021

Powell, J. L., Lewis, P. A., Dunbar, R. I. M., García-Fiñana, M., \& Roberts, N. (2010). Orbital prefrontal cortex volume correlates with social cognitive competence. Neuropsychologia, 48(12), 3554-3562. https://doi.org/10.1016/j.neuropsychologia.2010.08.004

Premack, D., \& Woodruff, G. (1978). Does the chimpanzee have a theory of mind? Behavioral and Brain Sciences, 1(4), 515-526. https://doi.org/10.1017/s0140525x00076512

Reis, H. T., \& Shaver, P. (1988). Intimacy as an interpersonal process. In S. W. Duck, D. F. Hay, S. E. Hobfoll, W. Ickes, \& B. M. Montgomery (Eds.), Handbook of personal relationships: Theory, research and interventions (pp. 367-389). John Wiley \& Sons Ltd. https://doi.org/10.4324/9780203732496-5

Richards, G., Baron-Cohen, S., Stokes, H., Warrier, V., Mellor, B., Winspear, E., Davies, J., Gee, L., \& Galvin, J. (2020). Assortative mating, autistic traits, empathizing, and systemizing. BioR $\chi i v$. https://doi.org/10.1101/2020.10.28.358895

Ronald, A., Happé, F., Hughes, C., \& Plomin, R. (2005). Nice and nasty theory of mind in preschool children: Nature and nurture. Social Development, 14(4), 664-684. https://doi.org/10.1111/j.1467-9507.2005.00323.x

Saroglou, V., \& Galand, P. (2004). Identities, values, and religion: A study among Muslim, other immigrant, and native Belgian young adults after the 9/11 attacks. Identity: An International Journal of Theory and Research, 4(2), 97-132. https://doi.org/10.1207/s1532706xid0402_1

Saroglou, V., \& Muñoz-García, A. (2008). Individual differences in religion and spirituality: An issue of personality traits and/or values. Journal for the Scientific Study of Religion, 47(1), 83-101. https://doi.org/10.1111/j.1468-5906.2008.00393.x

Shamay-Tsoory, S. G., \& Aharon-Peretz, J. (2007). Dissociable prefrontal networks for cognitive and affective theory of mind: A lesion study. Neuropsychologia, 45(13), 3054-3067. https://doi.org/10.1016/j.neuropsychologia.2007.05.021

Sharp, C. (2008). Theory of mind and conduct problems in children: Deficits in reading the "emotions of the eyes." Cognition and Emotion, 22(6), 1149-1158. https://doi.org/10.1080/02699930701667586

Śmieja, M., \& Stolarski, M. (2018). Assortative mating for emotional intelligence. Current Psychology, 37(1), 180-187. https://doi.org/10.1007/s12144-016-9501-8

Smith, L., Heaven, P. C. L., \& Ciarrochi, J. (2008). Trait emotional intelligence, conflict communication patterns, and relationship satisfaction. Personality and Individual Differences, 44(6), 1314-1325. https://doi.org/10.1016/j.paid.2007.11.024

Smith, R. L., \& Rose, A. J. (2011). The "cost of caring” in youths' friendships: Considering associations among social perspective-taking, co-rumination, and empathetic distress. 
Developmental Psychology, 47(6), 1792-1803. https://doi.org/10.1037/a0025309

Stiller, J., \& Dunbar, R. I. M. (2007). Perspective-taking and memory capacity predict social network size. Social Networks, 29(1), 93-104. https://doi.org/10.1016/j.socnet.2006.04.001

Sutton, J., Smith, P. K., \& Swettenham, J. (1999). Social cognition and bullying: Social inadequacy or skilled manipulation? British Journal of Developmental Psychology, 17(3), 435-450. https://doi.org/10.1348/026151099165384

Tager-Flusberg, H., \& Sullivan, K. (2000). A componential view of theory of mind: Evidence from Williams syndrome. Cognition, 76(1), 59-89. https://doi.org/10.1016/S00100277(00)00069-X

Tognetti, A., Berticat, C., Raymond, M., \& Faurie, C. (2014). Assortative mating based on cooperativeness and generosity. Journal of Evolutionary Biology, 27(5), 975-981. https://doi.org/10.1111/jeb.12346

Virkud, Y. V., Todd, R. D., Abbacchi, A. M., Zhang, Y., \& Constantino, J. N. (2009). Familial aggregation of quantitative autistic traits in multiplex versus simplex autism. American Journal of Medical Genetics, Part B: Neuropsychiatric Genetics, 150B(3), 328-334. https://doi.org/10.1002/ajmg.b.30810

Wainer, A. L., Block, N., Donnellan, M. B., \& Ingersoll, B. (2013). The broader autism phenotype and friendships in non-clinical dyads. Journal of Autism and Developmental Disorders, 43(10), 2418-2425. https://doi.org/10.1007/s10803-013-1789-8

Wu, R., Liu, Z., Guo, Q., Cai, M., \& Zhou, J. (2020). Couple similarity on personality, moral identity and spirituality predict life satisfaction of spouses and their offspring. Journal of Happiness Studies, 21(3), 1037-1058. https://doi.org/10.1007/s10902-019-00108-8 